\title{
Continuous functions on products of topological spaces
}

\section{J. Gerlits (Budapest)}

Abstract. Let $f$ be a continuous function from the product space $X=\prod\left\{X_{i} ; i \in I\right\}$ onto the $T_{2}$-space $Y$. Well-known results of Miščnko, Engelking and others state that under certain conditions $f$ depends only on few coordinates. In section 2 of the paper some generalizations of these theorems are given; e.g. if the cardinal $\alpha>\omega$ is a caliber for $X$ and $Y$ does not contain these theorems are given; e.g. if the cardinal $\alpha>\omega$ is a caliber for $X$ and $Y$ does not contain
topologically the Cantor cube of weight $\alpha$ then $f$ depends on $<\alpha$ coordinates (Corollary 3). In topologically the Cantor cube of weight $\alpha$ the
section 3 local-type variants are considered.

1. Let $\left\{X_{i} ; i \in I\right\}$ be a family of topological spaces, $X=\prod\left\{X_{i} ; i \in I\right\}$ the topological product and $f: X \rightarrow Y$ a mapping onto the $T_{2}$-space $Y$.

A well-known theorem of A. Miščenko [8] asserts that if $\alpha^{+}$is a caliber for $X$ and $\psi Y \leqslant \alpha\left({ }^{1}\right)$ then $f$ depends at most on $\alpha$ coordinates.

Our main aim in this paper is to prove a generalization of this theorem. Some applications, to derive a generalization of a theorem of R. Engelking [4] and a new result for dyadic compacta, will be given.

We shall use the usual set-theoretic notions; cardinals are identified with initial ordinals. All undefined terms can be found in [3] or [7].

In the sequel we shall need the following definitions and theorems.

Definition (N. Sanin [10]). A cardinality $\alpha$ is said to be a caliber for the topological space $X$ if given any sequence $\left\{G_{\xi} ; \xi<\alpha\right\}$ of non-empty open sets in $X$, there exists a set $A \subset \alpha,|A|=\alpha$ with $\bigcap\left\{G_{\xi} ; \xi \in A\right\} \neq \varnothing$.

(1) If $R$ is a topological space, $x \in R, A \subset R, x \in \bar{A}$,

$$
\begin{aligned}
y(x, R) & =\min \{|\mathfrak{S}| ; \mathcal{S} \text { is an open system, } \bigcap \mathcal{S}=\{x\}\}, \\
\chi(x, R) & =\min \{|\mathcal{S}| ; \mathcal{S} \text { is a nbd-base of } x \text { in } R\}, \\
a(x, A) & =\min \{|B| ; B \subset A, x \in \bar{B}\}, \\
t(x, R) & =\sup \{a(x, A) ; A \subset R, x \in \bar{A}\} \\
t(R) & =\sup \{t(x, R) ; x \in R\}
\end{aligned}
$$

$(R)$ is the tightness of the space $R$. 
THEOREM (N. Sanin [10]). Suppose $\alpha$ is a cardinality, $\operatorname{cf}(\alpha)>\omega$ and $\left\{X_{i} ; i \in I\right\}$ is a family of topological spaces, $w\left(X_{i}\right)<\operatorname{cf}(\alpha)$ for $i \in I$; then $\alpha$ is a caliber for the product space $X=\prod\left\{X_{i} ; i \in I\right\}$.

THEOREM (A. Hajnal [6]). Suppose $H$ is a set and $\varphi: H \rightarrow P(H)$ is a set-mapping (that is, for each element $x$ of $H, \varphi(x)$ is a subset of $H$ ). If there exists a cardinality $\beta$ with $|\varphi(x)|<\beta<|H|$ for each $x \in H$, then there exists a set $F \subset H .|F|=|H|$ such that $F$ is "free" with respect to $\varphi$, i.e., $\varphi(x) \cap F \subset\{x\}$ for each $x \in F$.

We shall use this theorem only in the special case when $\beta=\omega$; that was first proved by S. Piccard [9].

2. Defintrion 1. Let $\left\{X_{i}: i \in I\right\}$ be a family of sets, $X=\prod\left\{X_{i} ; i \in I\right\}$, $A \subset X, f$ be a function from $X$ to the set $Y$. The set $J \subset I$ determines $f$ in $A$ if $p \in A$, $q \in X, p|J=q| J$ implies $f(p)=f(q)$.

Denote by $\operatorname{ord}(f, A)=\min \{|J| ; J \subset I, J$ determines $f$ in $A\}$. Note that if $A=X$ then $J$ determines $f$ in $X$ iff $f$ depends only on the coordinates in $J$.

Denote by $D$ the two-point discrete space.

Now we can formulate our main result.

Theorem 2. Let $\left\{X_{i} ; i \in I\right\}$ be a family of topological spaces, $X=\prod\left\{X_{i} ; i \in I\right\}$, $f: X \rightarrow Y$ a mapping to the $T_{2}$-space $Y, A \subset X, \alpha>\omega$ a cardinality. If $\alpha$ is a caliber for the subspace $A$ and $\operatorname{ord}(f, A) \geqslant \alpha$, then there exists a subspace $C \subset X, C \cap A \neq \emptyset$ such that $C$ is homeomorphic with $D^{\alpha}$ and $f \mid C$ is a homeomorphism.

Proof. An elementary open set in $X$ is a set $U=\prod\left\{U_{i} ; i \in I\right\}$ where $U_{i} \subset X_{i}$ is an open set in $X_{i}$ for $i \in I$ and $I(U)=\left\{i \in I ; U_{i} \neq X_{i}\right\}$ is finite.

Suppose $\alpha>\omega$ is a caliber for $A \subset X$ and $\operatorname{ord}(f, A) \geqslant \alpha$. We shall define by transfinite induction a sequence $\left\{\left\langle p_{\xi}, q_{\xi}, U_{\xi}^{0}, U_{\xi}^{1}\right\rangle ; \xi<\alpha\right\}$.

Suppose $\varrho<\alpha$ and for $\xi<\varrho$ are defined $p_{\xi}, q_{\xi}, U_{\xi}^{0}, U_{\xi}^{1}$ with

(i) $p_{\xi} \in A, q_{\xi} \in X, U_{\xi}^{0}$ and $U_{\xi}^{1}$ are elementary open sets,

(ii) $p_{\xi} \in U_{\xi}^{0}, q_{\xi} \in U_{\xi}^{1}, I\left(U_{\xi}^{0}\right)=I\left(U_{\xi}^{1}\right)=I_{\xi}$,

(iii) if $\eta<\xi<Q$ then $p_{\xi}\left|I_{\eta}=q_{\xi}\right| I_{\eta}$,

(iv) if $i \in I$ and $p_{\xi}(i)=q_{\xi}(i)$, then $\pi_{i}\left(U_{\xi}^{\ominus}\right)=\pi_{i}\left(U_{\xi}^{1}\right)$,

(v) $f\left(U_{\xi}^{0}\right) \cap f\left(U_{\xi}^{1}\right)=\varnothing$.

Put $J=\bigcup\left\{I_{\xi} ; \xi<\varrho\right\}$; now $|J| \leqslant \varrho \cdot \omega<\alpha$.

By $\operatorname{ord}(f, A) \geqslant \alpha$ there exist two points $p_{Q}, q_{Q}, p_{Q} \in A, q_{Q} \in X, p_{Q}\left|J=q_{Q}\right| J$ with $f\left(p_{Q}\right) \neq f\left(q_{Q}\right)$.

The space $Y$ is $T_{2}$ and $f$ is continuous so there exist two elementary open sets $U_{a}^{0}, U_{\varrho}^{1}$ with $p_{Q} \in U_{Q}^{0}, q_{Q} \in U_{Q}^{1}, f\left(U_{Q}^{0}\right) \cap f\left(U_{Q}^{1}\right)=\varnothing$. Evidently we can suppose that $I\left(U_{e}^{0}\right)=I\left(U_{e}^{1}\right)=I_{e}$ and if $i \in I_{e}, p_{e}(i)=q_{e}(i)$, then $\pi_{i}\left(U_{\xi}^{0}\right)=\pi_{i}\left(U_{e}^{1}\right)$.

It is now very easy to check that the sequence $\left\{\left\langle p_{\xi}, q_{\xi}, U_{\xi}^{0}, U_{\xi}^{1}\right\rangle, \xi<\varrho+1\right\}$ satisfies our conditions (i)-(v).

Using now that $\alpha$ is a caliber for $A$ and $U_{\xi} \cap A \neq \varnothing$ for $\xi<\alpha$, we can assume that $p \in A \cap \cap\left\{U_{\xi}^{0} ; \xi<\alpha\right\}$.

For each $\xi<\alpha$ put $J_{\xi}=\left\{i \in I ; \pi_{i}\left(U_{\xi}^{0}\right) \neq \pi_{i}\left(U_{\xi}^{1}\right)\right\}$. Evidently, $J_{\xi} \subset I_{\xi}$. If $\eta<\xi<\alpha$, then, by (iii) and (iv), $J_{\xi} \cap I_{\eta}=\varnothing$; hence the sets $\left\{J_{\xi} ; \xi<\alpha\right\}$ are pairwise disjoint. By (v), the sets $J_{\xi}$ are non-empty $(\xi<\alpha)$.

If $\xi<\alpha$, put $\varphi(\xi)=\left\{\eta<\alpha ; J_{\eta} \cap I_{\xi} \neq \varnothing\right\}$.

The set $I_{\xi}$ is finite and the $J_{\eta}$ 's are disjoint, hence $|\varphi(\xi)|<\omega<\alpha$ for each $\xi<\alpha$. Using now Hajnal's theorem on set-mapping, we can suppose that

$$
J_{\xi} \cap I_{\eta} \neq \varnothing \quad \text { iff } \quad \xi=\eta(\xi<\alpha, \eta<\alpha) .
$$

Let now $g$ be the following function from $D^{\alpha}$ into $X$ : if $x \in D^{\alpha}, i \in I$ put

$$
g(x)(i)= \begin{cases}q_{\xi}(i) & \text { if } i \in J_{\xi} \text { and } x(\xi)=1 \\ p(i) & \text { otherwise. }\end{cases}
$$

The definition of $g$ is meaningful because the sets $J_{\xi}$ are disjoint. The function $g$ is continuous; indeed, it is enough to prove that $\pi_{i} \circ g$ is continuous for each $i \in I$.

If $i \in I-J_{\xi}$ for each $\xi<\alpha$, then $\pi_{i} \circ g$ is constant; if $i \in J_{\xi}$ for an ordinal $\xi<\alpha$, then $\pi_{i} \circ g$ is constant on two complementary clopen sets of $D^{\alpha}$.

We assert now that for each $x \in D^{\alpha}$ and $\xi<\alpha \quad g(x) \in U_{\xi}^{x(\xi)}$.

Note that if $i \in I-J_{\xi}$ or $\varepsilon=0$, then $p(i) \in \pi_{i}\left(U_{\xi}^{z}\right)$. Indeed, $p \in U_{\xi}^{0}$ for each $\xi<\alpha$; on the other hand, if $i \in I-J_{\xi}$, then $p(i) \in \pi_{i}\left(U_{\xi}^{0}\right)=\pi_{i}\left(U_{\xi}^{1}\right)$ by the definition of $J_{\xi}$. Now, we have to prove that for each $i \in I, g(x)(i) \in \pi_{i}\left(U_{\xi}^{x(\xi)}\right)$. This is evidently true if $i \notin I_{\xi}$, because, then $\pi_{i}\left(U_{\xi}^{x(\xi)}\right)=X_{i}$. If $i \in I_{\xi}-J_{\xi}$, then, by $(*), i \notin J_{\eta}$ for each $\eta<\alpha$ hence $g(x)(i)=p(i) \in \pi_{i}\left(U_{\xi}^{x(\xi)}\right)$. Finally, let $i \in J_{\xi}$. If $x(\xi)=0, g(x)(i)$ $=p(i) \in \pi_{i}\left(U_{\xi}^{x(\xi)}\right)$; if $x(\xi)=1, g(x)(i)=q_{\xi}(i)$ and $q_{\xi} \in U_{\xi}^{1}$ so $g(x)(i) \in \pi_{i}\left(U_{\xi}^{x(\xi)}\right)$.

Now, if $x, y \in D^{\alpha}, x \neq y$, then there exists a $\xi<\alpha$ with $x(\xi) \neq y(\xi)$. Hence $f\left(g(x) \in f\left(U_{\xi}^{x(\xi)}\right), \quad f(g(y)) \in f\left(U_{\xi}^{y(\xi)}\right)\right.$ and $f\left(U_{\xi}^{x(\xi)}\right) \cap f\left(U_{\xi}^{y(\xi)}\right)=\varnothing \quad$ by $(\mathrm{v})$, so $f(g(x)) \neq f(g(y))$.

The space $Y$ is $T_{2}$ consequently $h=f \circ g$ is a homeomorphic embedding of $D^{\alpha}$ into $Y$. Put $K=h\left(D^{\alpha}\right) \subset Y, C=g\left(D^{\alpha}\right) \subset X$. Now $h=f \circ g$ is a homeomorphic mapping from $D^{\alpha}$ onto $K$ and $g$ and $f$ are continuous; so $g$ is a homeomorphism of $D^{\alpha}$ onto $\dot{C}$

Applying the theorem for the case $A=X$, we get the promised generalization of the theorem of A. Miščenko:

COROLlaRY 3. Let the cardinality $\alpha>\omega$ be a caliber for the product space $X=\prod\left\{X_{i} ; i \in I\right\}, f: X \rightarrow Y$ a mapping into the $T_{2}$-space $Y$. If $Y$ does not contain a topological copy of $D^{\alpha}$, then $f$ depends on $<\alpha$ coordinates.

If $A=\{p\}$, then each cardinality $\alpha>\omega$ is a caliber for $A$, hence

Corollary 4. Let $X=\prod\left\{X_{i} ; i \in I\right\}, f: X \rightarrow Y$ a mapping onto the $T_{2}$-space $Y$, $p \in X$. If $\operatorname{ord}(f, p) \geqslant \alpha>\omega$, then there exists a set $C, p \in C \subset X$ such that $C$ is homeomorphic with $D^{\alpha}$ and $f \mid C$ is a homeomorphism.

The following lemma will be useful for us.

Lemma 5. Let $X=\prod\left\{X_{i} ; i \in I\right\}$ be the topological product of the spaces $X_{i}$, $w\left(X_{i}\right)<\alpha$ for each $i \in I$, where $\alpha>\omega$ is a regular cardinality. Suppose $A \subset X$ and for 
each $p \in A$ there exists a set $J=J(p) \subset I,|J|<\alpha$ such that $q \in X, p|J=q| J$ implies $q \in A$. Then $\alpha$ is a caliber for the subspace $A$.

Proof. For each $i \in I$ choose a base $\mathfrak{B}$ in $X_{i},\left|\mathfrak{B}_{i}\right|<\alpha, X_{i} \in \mathfrak{B}_{i}$. A set $U \subset X$ is a basic open set if $U=\prod\left\{U_{i} ; i \in I\right\}, U_{i} \in \mathfrak{B}_{i}$ for $i \in I$ and $I(U)=\left\{i \in I ; U_{i} \neq X_{i}\right\}$ is finite. It is enough to prove that if $\left\{U_{s} ; s \in S\right\}$ is a family of basic open sets in $X$ with $|S|=\alpha$ and $U_{s} \cap A \neq \varnothing$ for $s \in S$, then there exists a set $S^{\prime} \subset S,\left|S^{\prime}\right|=\alpha$ such that $\cap\left\{U_{s} ; s \in S^{\prime}\right\} \cap A \neq \varnothing$

By the Erdös-Radó theorem (see e.g. [7] A.2) we can suppose that the system $\left\{I\left(U_{s}\right) ; s \in S\right\}$ is a quasi-disjoint family; i.e., that for $s, s^{\prime} \in S, s \neq s^{\prime}$ $I\left(U_{s}\right) \cap I\left(U_{s^{\prime}}\right)=K$ holds. The set $K \subset I$ is finite and $\left|\mathfrak{B}_{i}\right|<\alpha$ for each $i \in I$, hence we can assume that for each $i \in K$ and for each $s \in S \pi_{i}\left(U_{s}\right)=G_{i}$.

Choose now an arbitrary $s_{0} \in S$ and $p_{0} \in A \cap U_{s_{0}}$. In accordance with our conditions there exists a set $J \subset I,|J|<\alpha$ such that $q \in X, q\left|J=p_{0}\right| J$ implies $q \in A$. The members of the family $\left\{I\left(U_{s}\right)-K ; s \in S\right\}$ are pairwise disjoint hence the set $S^{\prime}=\left\{s \in S, I\left(U_{s}\right) \cap J \subset K\right\}$ has cardinality $\alpha$. Select a point $q_{s} \in U_{s}$ for $s \in S^{\prime}$ and let $q \in X$ be the point

$$
q(i)= \begin{cases}q_{s}(i) & \text { if } i \in I\left(U_{s}\right)-K, s \in S^{\prime} \\ p_{0}(i) & \text { otherwise. }\end{cases}
$$

This is a meaningful definition because the sets $\left\{I\left(U_{s}\right)-K ;, s \in S^{\prime}\right\}$ are pair wise disjoint. Now $q\left|J=p_{0}\right| J$ so $q \in A$; on the other hand, evidently $q \in \cap\left\{U_{s}\right.$; $\left.s \in S^{\prime}\right\}$.

Now, let $X=\prod\left\{X_{i} ; i \in I\right\}$ be a product of topological spaces, $w\left(X_{i}\right)<\alpha$ $(i \in I), \alpha>\omega$ a regular cardinality and $f: X \rightarrow Y$ a mapping onto the $T_{2}-$ space $Y$. Denote by $H$ the set of those points $y \in Y$, for which it does not exist a compact set $C$, homeomorphic with $D^{\alpha}, y \in C \subset Y$. (For example, if the topological character of $y$ in $Y$ is less than $\alpha$, then $y \in H$.) Put $A=f^{-1}(H)$; evidently, it does not exist a set $C \subset X, C \cap A \neq \varnothing, C$ homeomorphic with $D^{\alpha}$ such that $f \mid C$ is a homeomorphism. This shows, by Corollary 4 , that for each $p \in A$ ord $(f, p)<\alpha$ and hence the conditions of Lemma 5 are satisfied, thus $\alpha$ is a caliber for $A$. Applying now Theorem 2, we conclude that $\operatorname{ord}(f, A)<\alpha$ and so there exists a set $J \subset I,|J|<\alpha$ such that $p \in A, q \in X, p|J=q| J$ implies $f(q)=f(p) \in H$ hence $q \in A$. This shows that $A=\pi_{J}^{-1}(B)$ where $\pi_{J}$ is the projection on the partial product

$$
X_{J}=\prod\left\{X_{i} ; i \in J\right\}
$$

and $B \subset X_{J}$. Moreover, $f \mid A$ depends only on the coordinates in $J$

Using that $Y$ is a $T_{2}$-space we immediately obtain $f \mid \bar{A}$ depends only on the coordinates of $J$, too. Hence we can conclude that if $A$ is dense in $X$, then $f$ depends only on $<\alpha$ coordinates, sharpening thus a theorem of R. Engelking [4]:

THEOREM 6. Let $f$ be a mapping from the topological product $X=\prod\left\{X_{i} ; i \in I\right\}$ of spaces $X_{i}$ with $w\left(X_{i}\right)<\alpha$ to a $T_{2}$-space $Y$, where $\alpha>\omega$ is a regular cardinality.
If $f$ depends on $\geqslant \alpha$ coordinates then for each $Q \subset X$ dense set in $X$ there exists a point $q \in Q$ and a space $C$ homeomorphic with $D^{\alpha}$ such that $f(q) \in C \subset Y$.

If the spaces $X_{i}$ are compact spaces, then the conclusion is simpler

THEOREM 7. Let $f$ be a mapping from the topological product $X=\prod\left\{X_{i} ; i \in I\right\}$ of compact spaces with $w\left(X_{i}\right)<\alpha$ onto a $T_{2}$-space $Y$, where $\alpha>\omega$ is a regular cardinality. If $w(Y) \geqslant \alpha$, then for each $Q \subset Y$ dense set there exists a subspace $C \subset Y$ homeomorphic with $D^{\alpha}, C \cap Q \neq \varnothing$.

Proof. Indeed, if the above set $H$ is dense in $Y$ then $f(\bar{A})=Y$ because $f$ is now a closed mapping. But evidently $\bar{A}=\overline{\pi_{J}^{-1}(B)}=\pi_{J}^{-1}(\bar{B})$ and so $Y=\left(f \circ \pi_{J}^{-1}\right)(\bar{B})$. Surely $w(\bar{B}) \leqslant w\left(X_{J}\right) \leqslant \sum\left\{w\left(X_{i}\right) ; i \in J\right\}<\alpha$ ( $\alpha$ is regular) hence by a theorem of Arhangel'skǐ (see e.g. [3] Corollary 2 to Theorem 3.1.11) $w(Y)<\alpha$ too.

This is a sharpening of a theorem of R. Engelking [5].

3. In this section we are investigating the local version of the issue of the preceding section.

Let $\alpha>\omega$ be a regular cardinality, $w\left(X_{i}\right)<\alpha(i \in I), X=\prod\left\{X_{i} ; i \in I\right\}, f: X \rightarrow Y$ a continuous mapping onto the $T_{2}$-space $Y$.

Now, a typical "local problem" is the following: suppose $y \in Y, \chi(y, Y) \geqslant \alpha$; can we assert that there exists a set $C, y \in C \subset Y, C$ homeomorphic with $D^{\alpha}$ ?

The answer to this question is in general negative; indeed if $\alpha=\omega_{1}$, it is easy to construct a $T_{5}$ topological space $Y$ with $|Y|=\omega, \chi(y, Y)=\omega_{1}$ for each $y \in Y\left({ }^{2}\right)$; this space $Y$ is the continuous image of the discrete countable space $\boldsymbol{N}_{\text {, }}$ $w(N)=\omega<\omega_{1}$. What is more, the answer is negative even if we assume only that $\psi(y, Y) \geqslant \alpha=\omega_{1}$.

EXAMPLE 8 . The basic set of our space $Y$ will be $N^{\omega_{1}} \cup\{p\}, p \notin N^{\omega_{1}}, N^{\omega_{1}}$ is an open subspace of $Y$ and $\psi(p, Y)=\omega_{1}$. The existence of such a space is guaranteed by the fact that $N^{\omega_{1}}$ is a zerodimensional non-Lindelöf space but it can also be constructed directly. For example, a neighbourhood-base of the point $p$ is the system

$$
\left\{U_{T}(p) ; T \subset \omega_{1},|T|<\omega\right\}
$$

where

$$
U_{T}(p)=\left\{q \in N^{\omega_{1}} ; q(i) \neq q(j) \text { if } i, j \in T, i \neq j\right\} \cup\{p\} .
$$

Evidently $Y$ is the continuous image of the product space $N^{\omega_{1}}, w(N)=\omega<\omega_{1}$, $\psi(y, Y)=\omega_{1}$ for each $y \in Y$.

We assert that does not exist a set $C$ homeomorphic with $D^{\omega_{1}}, p \in C \subset Y$. Indeed, suppose on the contrary that $\varphi: D^{\omega_{1}} \rightarrow Y$ is an embedding, $\varphi(\mathbf{1})=p$.

Put

$$
\begin{aligned}
& \Sigma=\left\{q \in D^{\omega_{1}} ;\left|\left\{\xi<\omega_{1} ; q(\xi)=1\right\}\right| \leqslant \omega\right\}, \\
& A=f(\Sigma) .
\end{aligned}
$$

Evidently, $A \subset N^{\omega_{1}}, p \in \bar{A}$, moreover, if $B \subset A,|B| \leqslant \omega$, then $\bar{B} \subset A, \bar{B}$ is a compact 
set. Now, for each $\xi<\omega_{1}, \pi_{\xi}(A)$ is a finite subset of $N$; indeed, otherwise we should choose a countable set $B \subset A$ with $\pi_{\xi}(B)$ infinite and this contradicts to the fact that $\pi_{\xi}(\bar{B})$ is compact in the discrete space $N$. Put $K=\prod\left\{\pi_{\xi}(A) ; \xi<\omega_{1}\right\}$. Now $A \subset K_{\text {a }}$

$K$ is a compact set in $N^{\omega_{1}}$ hence $p \notin \bar{A}$, a contradiction.

Nevertheless, the following theorem can be asserted.

THEOREM 9. Let $f$ be a continuous function from the product space

$$
X=\prod\left\{X_{i} ; i \in I\right\}
$$

of spaces $X_{i}$ with $w\left(X_{i}\right)<\alpha$ onto the $T_{2}$-space $Y$. If $\alpha>\omega$ is a regular cardinality and $y \in Y$ with $\psi(y, Y) \geqslant \alpha$, then there exists a set $A \subset Y$ such that $y \notin A, a(y, A) \geqslant \alpha$.

Proof. Put $F=f^{-1}(y), G=X-F$. Denote by

$$
\mathfrak{H}=\{A \subset G ; \psi(y, f(A) \cup\{y\}) \geqslant \alpha\} .
$$

Evidently $G \in \mathfrak{A}$; moreover, if $A \in \mathfrak{A}, A=\bigcup\left\{A_{\xi} ; \xi<\beta\right\}$ and $\beta<\alpha$ then there exists a $\xi<\beta$ with $A_{\xi} \in \mathfrak{A}$. Note that if $A \in \mathfrak{A}$ then $y \in \overline{f(A)}$. Choose now a base $\mathfrak{B}_{i}$ in $X_{i}$ with $\left|\mathfrak{B}_{i}\right|<\alpha, X_{i} \in \mathfrak{B}_{i}(i \in I)$. A basic open set in $X$ is a set $U=\prod\left\{U_{i} ; i \in I\right\}$ with $U_{i} \in \mathfrak{B}_{i}$ for $i \in I$ and $I(U)=\left\{i \in I ; U_{i} \neq X_{i}\right\}$ finite.

Making use of the fact that $Y$ is $T_{2}$ and $f$ continuous we get a cover $\mathfrak{H}$ of $G$ of basic open sets such that

$$
\overline{f(U)} \subset Y-\{y\} \quad \text { for each } U \in \mathfrak{H} .
$$

Denote by

$$
\sigma=\{\mathfrak{B} \subset \mathfrak{U} ; \quad U\{V ; V \in \mathfrak{B}\} \in \mathfrak{U}\} .
$$

Evidently $\mathfrak{H} \in \sigma$ and if

$$
\mathfrak{B} \in \sigma, \quad \mathfrak{B}=\bigcup\left\{\mathfrak{B}_{\xi} ; \xi<\beta\right\}
$$

and $\beta<\alpha$ then there exists a $\xi<\beta$ with $\mathfrak{B}_{\xi} \in \sigma$.

Put now

$$
\mathfrak{U}_{n}=\{U \in \mathfrak{U} ;|I(U)| \leqslant n\} \quad(n<\omega) .
$$

$\mathfrak{U}=\bigcup\left\{\mathfrak{H}_{n} ; n<\omega\right.$ and $\omega<\alpha$ implies $\mathfrak{U}_{m} \in \sigma$ for a suitable $m<\omega$.

Let now $r$ be the maximal integer such that there exists a set $T \subset I,|T|=r$ with

$$
\mathfrak{U}(T)=\left\{U \in \mathfrak{I}_{m} ; T \subset I(U)\right\} \in \sigma \quad(0 \leqslant r \leqslant m) .
$$

Select $T_{0} \subset I,\left|T_{0}\right|=r$ with $\mathfrak{U}\left(T_{0}\right) \in \sigma$. Using now (*) and that $\left|\mathfrak{B}_{t}\right|<\alpha$ for $t \in T_{0}$ we get a family $\mathfrak{B} \subset \mathfrak{U}\left(T_{0}\right), \mathfrak{B} \in \sigma$ with $\pi_{t}(U)=B_{t}$ for each $U \in \mathfrak{B}, t \in T_{0}$.

If $i \in I-T_{0}$, then, by the maximality of $r, \mathfrak{B}_{i}=\{U \in \mathfrak{B} ; i \in I(U)\} \notin \sigma$. More generally, if $J \subset I-T_{0},|J|<\alpha$, then, by (*), if $\mathfrak{B}_{J}=\bigcup\left\{\mathfrak{N}_{i} ; i \in J\right\}, \mathfrak{B}_{J} \notin \sigma$ hence there exists a set $U \in \mathfrak{B}$ with $I(U) \cap J=\varnothing$.

This shows that we can choose a family $\left\{U_{\xi} ; \xi<\alpha\right\} \subset \mathfrak{B}$ with $I\left(U_{\xi}\right) \cap I\left(U_{\eta}\right)=\varnothing$
Denote now by $\Sigma$ the set of those points in $X$ which are contained in all but

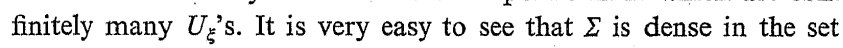

$$
\bigcap\left\{\pi_{i}^{-1}\left(B_{t}\right) ; t \in T_{0}\right\}
$$

hence $\overline{U\{U ; U \in \mathfrak{B}\}} \subset \bar{\Sigma}$. This implies, of course, that if $A=f(\Sigma)$ then $y \in \bar{A}$. On the other hand, if $P \subset \Sigma,|P|<\alpha$ then there exists a $\xi<\alpha$ with $P \subset U_{\xi}$ hence $f(P) \subset f\left(U_{\xi}\right)$. But $U_{\xi} \in \mathfrak{B} \subset \mathfrak{U}$ hence $y \notin \overline{f\left(U_{\xi}\right)}$ so $y \notin \overline{f(P)}$. This shows that $a(y, A) \geqslant \alpha$.

Assuming that "almost all" factor spaces $X_{i}$ are compact, we get a sharper result.

THEOREM 10. Let $f$ be a mapping from the topological product $\prod\left\{X_{i} ; i \in I\right\}$ of spaces with $w\left(X_{i}\right)<\alpha$ onto the $T_{2}$-space $Y$ where $\alpha>\omega$ is a regular cardinality. Suppose that the set $\left\{i \in I ; X_{i}\right.$ is not compact $\}$ has cardinality $<\alpha$ and $y \in Y$, $\psi(y, Y) \geqslant \alpha$; then there exists a space $C$, homeomorphic with $D^{\alpha}, y \in C \subset Y$.

Proof. Suppose it does not exist such a subset for the point $y \in Y$.

Denote by $A=f^{-1}(y) \subset X$. Exactly as in the proof of Theorem 6, we can prove that there exists a set $J \subset I,|J|<\alpha$ such that $A=\pi_{J}^{-1}(B)$ where $B \subset X_{J}$. Evidently we can also assume that if $i \in I-J$ then $X_{i}$ is compact. Now $A$ is a closed set in $X$ and so $B$ is also a closed set in $X_{J}$ because $\pi_{J}^{-1}(B)=A=\bar{A}=\pi_{J}^{-1}(\bar{B})$ hence $B=\bar{B}$.

Now $w\left(X_{J}\right)<\alpha$ hence there exists a base $\mathfrak{B}$ of $X_{J},|\mathfrak{B}|<\alpha$. For $B \in \mathfrak{B}$, put $F_{B}=\overline{f\left(\pi_{j}^{-1}(B)\right)} \subset Y$ and put $\mathfrak{Q}=\left\{B \in \mathfrak{B} ; y \notin F_{B}\right\}$.

It is enough to prove that $Y-\{y\}=\bigcup\left\{F_{B} ; B \in \mathfrak{A}\right\}$ because then $\{y\}=\bigcap\left\{Y-F_{B} ; B \in \mathfrak{A}\right\}$ and $\psi(y, Y) \leqslant|\mathfrak{M}| \leqslant|\mathfrak{B}|<\alpha$.

Let $x \in Y-\{y\}$ and choose a point $p \in f^{-1}(x)$.

Put $p^{\prime}=\pi_{J}(p) \in X_{J}-B$. The set $C=\pi_{J}^{-1}\left(p^{1}\right) \subset X$ is compact and so $K=f(C) \subset Y$ is compact, too, $y \notin K, x \in K$. Select an open set $G \subset Y, K \subset G, y \notin \bar{G}$.

Using the compactness of the subspace $C$ we get a $B \in \mathfrak{B}$ with $p^{\prime} \in B$, $\pi_{J}^{-1}(B) \subset f^{-1}(G)$; now $C \subset \pi_{J}^{-1}(B)$.

So we proved $x \in F_{B} \subset \bar{G} \subset Y-\{y\}$ hence $B \in \mathfrak{A}$.

Using this theorem we can deduce a seemingly new result for dyadic compacta. (A dyadic compactum is a Housdorffic image of a product space $D^{I}$.)

THEOREM 11. Let $R$ be a dyadic compactum, $x \in R, \alpha>\omega$ a regular cardinality; then the following conditions are equivalent

a) $\chi(x, R) \geqslant \alpha$

b) there exists a set $A \subset R$ with $x \in \bar{A}, a(x, A) \geqslant \alpha$,

c) there exists a subspace $C \subset R, C$ homeomorphic with $D^{\alpha}, x \in C$.

Proof. a) $\rightarrow$ c). This follows from Theorem 8 because in a compact space $\psi(x, R)=\chi(x, R)$ for each point.

c) $\rightarrow$ b). Put $A=\left\{y \in D^{\alpha} ;|\{\xi<\alpha ; x(\xi)=y(\xi)\}|<\alpha\right.$ for $x \in D^{\alpha}$. It is very easy to see that $A$ works.

b) $\rightarrow$ a). Trivial. 
Corollary 12. Let $R$ be a dyadic compactum, $x \in R$; then $\chi(x, R)=t(x, R)$.

The "global" version of Corollary 12 (i.e. that for a dyadic compactum $R$ $\chi(R)=t(R)$ holds) is a well-known fact. By a theorem of A. Arhangel'skil and V. Ponomariov [1] $t(R)=w(R)$ for a dyadic compactum $R$; moreover, if $w(R)=\alpha$ and $\operatorname{cf}(\alpha)>\omega$, then there exists a point $x \in R$ and a set $A \subset R$, such that $x \in \bar{A}$ and $a(x, A) \geqslant \alpha$. So it is natural to guess that Theorem 11 remains true if we suppose only that $\operatorname{cf}(\alpha)>\omega$. The following example is a counterexample to this conjecture; it is a modification of a construction due to B. Efimov [2].

EXAMPLE 13. Let $\alpha>\omega$ be a singular cardinality, $\beta=\operatorname{cf}(\alpha)<\alpha$. Select a sequence $\left\{\alpha_{\xi} ; \xi<\beta\right\}$ with $\alpha=\sup \left\{\alpha_{\xi} ; \xi<\beta\right\}, \beta<\alpha_{\xi}<\alpha_{\eta}<\alpha$ for $\xi<\eta<\beta$. Put

$$
\begin{aligned}
\Phi & =\left\{p \in D^{\alpha} ; p \mid \beta \equiv 0\right\}, \\
\Phi_{\xi} & =\left\{p \in D^{\alpha} ; p(\xi)=1, p \mid \alpha_{\xi}-\{\xi\} \equiv 0\right\} \quad(\xi<\beta), \\
F & =\Phi \cup \cup\left\{\Phi_{\xi} ; \xi<\beta\right\} .
\end{aligned}
$$

Now $F \subset D^{\alpha}$ is a closed set. Indeed, if $p \in D^{\alpha}$ and for each $\xi<\beta p(\xi)=0$, then $p \in \Phi \subset F$. If there is an ordinal $\xi<\beta$ with $p(\xi)=1$ and $p \notin \Phi_{\xi}$ then there exists an $\eta<\alpha_{\xi}, \eta \neq \xi$ with $p(\eta)=1$; now $U=\pi_{\xi}^{-1}(1) \cap \pi_{\eta}^{-1}(1)$ is a neighbourhood of $p$ and $U \cap F=\varnothing$.

Denote now by $R$ the quotient space identifying the points of $F ; R$ is certainly a dyadic compactum; denote by $x$ the point $\varphi(F)$ of $R$ where $\varphi$ is the quotient mapping.

If $\xi<\beta$, let $A_{\xi}=\left\{p \in D^{\alpha} ; p(\xi)=1,|\{\eta<\alpha ; p(\eta)=0\}|<\omega\right\}$. Evidently $\bar{A}_{\xi} \supset \Phi_{\xi}$ but if $B \subset A_{\xi},|B|<\alpha_{\xi}$ then $\bar{B} \cap \Phi_{\xi}=\varnothing$. This shows that in $R x \in \bar{A}_{\xi}$ and $a\left(x, A_{\xi}\right) \geqslant \alpha_{\xi}$. Specially, $\chi(x, R) \geqslant \alpha_{\xi}$ for each $\xi<\beta$ hence $\chi(x, R)=\alpha$.

On the other hand, if $A \subset D^{\alpha}-F, \bar{A} \cap F \neq \varnothing$, let $p \in \bar{A} \cap F$. Suppose $p \in \Phi_{\xi}$, $\xi<\beta$. If $J \subset \alpha_{\xi}$ is a finite set, there exists a point $q_{J} \in A_{\xi}$ with $p\left|J=q_{J}\right| J$. Put $B=\left\{q_{J} ; J \subset \alpha_{\xi},|J|<\omega\right\}, C=\pi_{\alpha_{\xi}}(\bar{B}) \subset D^{\alpha_{\xi}}$.

Now, in $D^{\alpha_{\xi}}, p^{\prime}=\pi_{\alpha_{\xi}}(p)$ is in the closure of the compact set $C$ hence $p^{\prime} \in C$. This means that there exists a point $q \in \bar{B}$ with $q\left|\alpha_{\xi}=p\right| \alpha_{\xi}$ but then $q \in \Phi_{\xi}$ and hence $a(x, A) \leqslant \alpha_{\xi}<\alpha$ in $R$.

Quite similarly, if $p \in \Phi \cap \bar{A}$ then $a(p, A) \leqslant \beta<\alpha$ in $R$. Hence in $R$ if $A \subset R$ $x \in \bar{A}$ then $a(x, A)<\alpha$.

\section{References}

[1] A. V. Arhangel'skiř and V. Ponomariov, On dyadic bicompacta, Dokłady Akad. Nauk SSSR 182 (1968), pp. 993-996.

[2] B. Efimov, On the weighted structure of dyadic compacti, Vestnik Moscow Univ., Ser. I Mat. Meh. 2 (1964), pp. 3-11.

[3] R. Engelking, Outline of General Topology, Warszawa 1968.

[4] - On functions defined on Cartesian products, Fund. Math. 59 (1966), pp. 221-231.

[5] - Cartesian products and dyadic spaces, Fund. Math. 57 (1966), pp. 287-304.
[6] A. Hajnal, Proof of a conjecture of S. Ruziewicz, Fund. Math. 50 (1961-1962), pp. 123-128. [7] I. Juhász, Cardinal Functions in Topology, Amsterdam 1971.

[8] A. Miščenko, Some theorems on the topological product of spaces, Fund. Math. 58 (1966), pp. 259-284.

[9] S. Piccard, Solution d'un problème de la théorie des relations, Fund. Math. 2 (1937), pp. 197-202.

[10] N. Shanin, On the product of topological spaces, Trudy Inst. Steklova 24, Moscow 1948.

\section{MATHEMATICAL INSTTTUTE OF THE HUNGARIAN ACADEMY OF SCIENCES} Budapest, Hungary 